Pacific Journal of Mathematics

REAL ANALYTIC OPEN MAPS 


\section{REAL ANALYTIC OPEN MAPS}

\section{P. T. CHURCh AND J. G. TIMOURIAN}

Let $R$ and $C$ be the real and complex fields, respectively, and for $\zeta \in C$ let $\mathscr{R}(\zeta)$ be the real part of $\zeta$. If $f: M^{p+1} \rightarrow N^{p}$ is real analytic and open with $p \geqq 1$, then there is a closed subspace $X \subset M^{p+1}$ such that $\operatorname{dim} f(X) \leqq p-2$ and, for every $x \in M^{p+1}-X$, there is a natural number $d(x)$ with $f$ at $x$ locally topologically equivalent to the map

$$
\phi_{d(x)}: C \times R^{p-1} \longrightarrow R \times R^{p-1}
$$

defined by $\phi_{d(x)}\left(z, t_{1}, \cdots, t_{p-1}\right)=\left(\mathscr{R}\left(z^{\alpha(x)}\right), t_{1}, \cdots, t_{p-1}\right)$.

In [7] Nathan proved: If $f: M^{2} \rightarrow N^{1}$ is real analytic and open, then for every $x \in M^{2}$ there is a natural number $d(x)$ with $f$ at $x$ locally topologically equivalent to the map $\phi_{d(x)}: C \rightarrow R$ defined by $\phi_{d(x)}(z)=\mathscr{R}\left(z^{d(x)}\right)$. This is the case $p=1$ of the above theorem, but our proof is not a generalization of his.

Examples (see (3.3)) show that "topologically equivalent" cannot be replaced by "analytically equivalent" or even " $C{ }^{1}$ equivalent", $f$ real analytic cannot be replaced by $f C^{\infty}$ (but see (3.1)), an exceptional set $X$ with $\operatorname{dim} f(X) \geqq p-2$ is needed, and $\operatorname{dim} X$ may be $p-1$.

Conventions 1.2. We must assume that the reader has [2] at hand, and we follow its conventions. In particular we need [2; $(2.2)$, (2.4), (2.5), (2.6), (2.8), (2.9), (3.1), and (3.9)]. For $f: M^{n} \rightarrow N^{p}, B_{f}$ is the set of $x$ in $M^{n}$ at which $f$ is not locally topologically equivalent to the projection map $\rho: R^{n} \rightarrow R^{p}$. The symbol $\approx$ is read "is diffeomorphic to".

Definitions 1.3. $C$-analytic sets are defined in [2]. A $C$-analytic set is called $C$-irreducible $[9$, p. 155] if it is not the sum of two $C$ analytic subsets distinct from itself. Whitney and Bruhat $[9, \mathrm{p} .155$, Proposition 11] prove that any $C$-analytic set $V$ is uniquely the (countable) locally finite union of $C$-irreducible $C$-analytic subsets $V_{m}$, no one of which contains another. The $V_{m}$ are called the $C$-irreducible components of $V$. Conversely, any locally finite union of $C$-analytic sets is a $C$-analytic set [9, p. 154].

DeFinitions 1.4. Let $V$ be a complex analytic set of dimension $v$. There is a complex analytic subset $S \subset V$ such that $\operatorname{dim} S<v$ and $V-S$ is a complex analytic $v$-manifold [8, p. 500]. (The points of 
$V-S$ are called simple or regular.) Let $M$ be a complex analytic manifold, and let $T(M, p)$ for $p \in M$ be the tangent plane of $M$ at $p$. Suppose that for each $p \in M, v$-plane $T$, and sequence $\left\{q_{i}\right\} \subset V-S$ with $q_{i} \rightarrow p$ and $T\left(V, q_{i}\right) \rightarrow T$, we have $T(M, p) \subset T$; then $V$ is said to be a-regular over $M$. If $V$ and $M$ also satisfy another property (b-regular), then $V$ is said to be regular over $M[8, \mathrm{p} .540]$.

Lemma 1.5. (Whitney [7, p. 540, Lemma 19.3].) Suppose that $V$ and $W$ are complex analytic sets, and $\operatorname{dim} V>\operatorname{dim} W$. Then there is a complex analytic subset $S$ of $W$ such that $\operatorname{dim} S<\operatorname{dim} W$, each point of $W-S$ is simple, and $V$ is regular over the complex analytic manifold $W-S$.

\section{Analytic sets and maps.}

LEMma 2.1. Let $f: M^{n} \rightarrow N^{p}$ be $C^{\omega}$, and let $V \subset M^{n}$ be a nonempty $C$-analytic subset of $M^{n}$ with dimension $v$. Then

(a) (Whitney and Bruhat [9, p. 156, Proposition 13]), there is a $C$-analytic subset $S \subset V$ such that $\operatorname{dim} S \leqq v-1$ and $V-S$ is a $v$ dimensional $C^{\omega}$ submanifold of $M^{n}$;

(b) there is a C-analytic subset $E \subset V$ such that $V-E$ is a vdimensional $C^{\omega}$ submanifold of $M^{n}, f \mid(V-E)$ has constant rank $r$, and $\operatorname{dim} f(E) \leqq v-1$;

(c) $\operatorname{dim} f(V) \leqq \max \{v-1, r\} \leqq v$; and

(d) if $V$ is $C$-irreducible (1.3), then $\operatorname{dim} f(V) \leqq r$.

Proof. We use induction on $v$; if $v=0$, then $V$ is discrete and the results are trivial.

Let $V_{m}$ be the $C$-irreducible components of $V$ (1.3). According to $[2$, p. $22,(3.1)]$ there is a $C$-analytic subset $E_{m}$ of $V_{m}$ such that $\operatorname{dim} E_{m}<\operatorname{dim} V_{m}, V_{m}-E_{m}$ is a $C^{\omega}$ submanifold of $V_{m}$ with dimension $\operatorname{dim} V_{m}$, and $f \mid\left(V_{m}-E_{m}\right)$ has constant rank $r_{m}$. Let $r$ be the maximum $r_{m}$ for those $m$ with $\operatorname{dim} V_{m}=v$.

If (1) $\operatorname{dim} V_{m}<v$, or (2) $\operatorname{dim} V_{m}=v$ and $r_{m}<r$, let $F_{m}=V_{m}$; (3) otherwise, let $F_{m}=E_{m}$. Since the $V_{m}$ are locally finite, the $F_{m}$ are also. Let $S \subset V$ be the $C$-analytic subset given by (a). Then by inductive hypothesis (c), $\operatorname{dim} f(S)<v$ and $\operatorname{dim} f\left(F_{m}\right)<v$ in cases (1) and (3). In case (2) $\operatorname{dim} f\left(E_{m}\right)<v$ also, and, from the Rank Theorem [1, p. 155] applied to $f \mid\left(V_{m}-E_{m}\right), \operatorname{dim}\left(f\left(V_{m}-E_{m}\right)\right) \leqq r_{m}<r \leqq v$. Since each of $E_{m}$ and $\left(V_{m}-E_{m}\right)$ is the countable union of compact sets, $\operatorname{dim} f\left(V_{m}\right)<v$. Let $E=S \cup\left(\bigcup_{m} F_{m}\right)$. Then $\operatorname{dim} f(E)<v ;(b)$ results from the local finiteness of the $F_{m}$ and (1.3); and (again from the Rank Theorem) (c) is a corollary. 
Now suppose that $V$ is $C$-irreducible. Let $W$ be the set $E$ of (b), let $W_{m}$ be its $C$-irreducible components, and let $E_{m}$ be as given by (b) for $W_{m}$. If each $f \mid\left(W_{m}-E_{m}\right)$ has rank at most $r$, then $\operatorname{dim} f\left(W_{m}\right) \leqq r$ by inductive hypothesis, and (d) follows. Thus we may suppose that for some $W_{m}$ and $E_{m}$, call them $W$ and $E, f \mid(W-E)$ has rank greater than $r$.

Let $M^{*}, N^{*}, f^{*}, V^{*}, W^{*}$, and $(W-E)^{*}$ be complexifications (see e.g. [2, (2.4), (2.5), (2.6)]), where $M^{*}$ is small enough that $V^{*}$ is irreducible in $M^{*}$ [9, p. 155, Proposition 11 and p. 151, Corollary 2]. Let $E^{\prime} \subset V^{*}$ be as given by $[2,(3.1)]$ for $V^{*}$ and $f^{*}$, so that $f^{*} \mid\left(V^{*}-E^{\prime}\right)$ has constant rank $k$. By definition of $r, V$ has a simple point $x$ at which $f \mid V$ has rank $r$; thus $f^{*} \mid V^{*}$ has rank $r$ at $x$ also, so that $k \geqq r$. Since $\operatorname{dim} E^{\prime}<\operatorname{dim} V^{*}=v$ [9, p. 155, Proposition 12], $\operatorname{dim}\left(E^{\prime} \cap M^{n}\right)<v$; thus $k=r$.

Let $S^{*}$ be the analytic subset of $(W-E)^{*}$ given by (1.5) such that $V^{*}$ is regular over the manifold $X^{*}=(W-E)^{*}-S^{*}$ and let $q \in X^{*}$. Since $V^{*}$ is irreducible, the simple points of $V^{*}$ are dense in $V^{*}\left[5\right.$, p. 68, Corollary 2]. Thus $V^{*}=C l\left[V^{*}-E^{\prime}\right]$, so there exist $q_{i} \in V^{*}-E^{\prime}$ with $q_{i} \rightarrow q$. Let $T_{i}$ and $T$ be the tangent planes of $V^{*}-E^{\prime}$ at $q_{i}$ and of $X^{*}$ at $q$, respectively. Since the Grassman manifold $G$ of $v$-planes in $C^{n}$ is compact, there are $T^{\prime} \in G$ and a subsequence $T_{i(j)} \rightarrow T^{\prime}$, and since $V^{*}$ is $a$-regular over $X^{*}, T \subset T^{\prime}$. Now $f \mid\left(V^{*}-E^{\prime}\right)$ has rank $r$, while $f^{*} \mid X^{*}$ has rank greater than $r$, and a contradiction results.

Substantially the same proof yields the complex analog, where $C$-analytic is replaced by analytic. There is a unique minimal set $E$ satisfying (b), viz. the intersection of all sets $E$ satisfying (b).

Lemma 2.2. Let $f: K^{k} \times R^{p-1} \rightarrow R \times R^{p-1}(p \geqq 1)$ be a $C^{\omega}$ layer map (i.e., $\left.f\left(K^{k} \times\{t\}\right) \subset R \times\{t\}\right)$, let $f_{t}: K^{k} \rightarrow R$ be defined by $\left(f_{t}(x), t\right)=$ $f(x, t)$, and let $\Gamma \subset R_{p-1}(f)$ be a C-analytic subset with $\operatorname{dim} \Gamma \leqq p-1$. Then there is a C-analytic subset $\Delta \subset \Gamma$ such that $\operatorname{dim} f(\Delta) \leqq p-2$ and

$$
\operatorname{dim}\left((\Gamma-\Delta) \cap\left(K^{k} \times\{t\}\right)\right) \leqq 0
$$

for each $t \in R^{p-1}$.

Proof. Let $E \subset \Gamma$ and $r$ be as given by (2.1(b)). If $r<p-1$, then let $\Delta=\Gamma$; if $r=p-1$, let $\Delta=E$. In either case, $\operatorname{dim} f(\Delta) \leqq$ $p-2(2.1(\mathrm{c}))$. If $\Gamma-\Delta \neq \varnothing$, it is a $C^{\omega}(p-1)$-manifold, and $f \mid(\Gamma-\Delta)$ has rank $p-1$. Since $\Gamma \subset R_{p_{-1}}(f), R_{p-1}(f) \cap\left(K^{k} \times\{t\}\right)=R_{0}\left(f_{t}\right)$, and $\operatorname{dim}\left(f_{t}\left(R_{0}\left(f_{t}\right)\right)\right) \leqq 0$ (by Sard's Theorem [1, p. 156]), $\Gamma-\Delta$ is transverse to each $K^{k} \times\{t\}$ at each point of intersection. In other words, the inclusion map $i: \Gamma-\Delta \rightarrow K^{k} \times R^{p-1}$ is transverse regular on $K^{k} \times$ $\{t\}$, so by Thom's Transversality Theorem [1, p. 165] $i^{-1}\left(K^{k} \times\{t\}\right)=$ 
$(\Gamma-\Delta) \cap\left(K^{k}\{t\}\right)$ is a 0 -dimensional manifold.

Lemma 2.3. If $f: R^{2} \times R^{p-1} \rightarrow R \times R^{p-1}$ is an open $C^{\omega}$ layer map, then there is a closed subset $X \subset R^{2} \times R^{p-1}$ such that $\operatorname{dim} f(X) \leqq p-2$ and $\operatorname{dim}\left(\left(B_{f}-X\right) \cap f^{-1}(y, t)\right) \leqq 0$ for each $(y, t) \in R \times R^{p-1}$.

Proof. By the Rank Theorem [1, p. 155] $B_{f} \subset R_{p-1}(f)$. (*) It suffices to prove the theorem locally, i.e., to show that for each $(x, t) \in R_{p-1}(f)$, there are neighborhoods $P \approx R^{2}$ of $x$ and $Q \approx R^{p-1}$ of $t$ such that $f \mid P \times Q$ satisfies the conclusion.

Now $R_{p-1}(f)$ is a $C$-analytic set [2,(2.9)], and since $\operatorname{dim}\left(f\left(R_{p-1}(f)\right)\right) \leqq$ $p-1$ by Sard's Theorem [1, p. 156] and $f$ is open, $\operatorname{dim}\left(R_{p-1}(f)\right) \leqq p$. It is the union of its $C$-irreducible components $V_{m}$ with dimension $v_{m}$; let $E_{m}$ and $r_{m}$ be as given by (2.1(b)) (or [2, (3.1)]). Let $E$ be the union of the $C$-analytic subset $S \subset R_{p-1}(f)$ given by (2.1(a)), the $V_{m}$ for $v_{m}=r_{m}=p-1$, and the $E_{m}$ for $v_{m}=p$ and $r_{m}=p-1$, and let $F$ be the union of the $V_{m}$ with $r_{m} \leqq p-2$. Let $G \subset E$ be the $C$ analytic subset $\Delta$ given by (2.2) for $\Gamma=E$. Then $\operatorname{dim}(f(F \cup G)) \leqq$ $p-2(2.1(\mathrm{~d}))$, so we may define $X$ to contain $F \cup G$. Thus (see $\left({ }^{*}\right)$ ) we may consider only neighborhoods $P \times Q$ disjoint from $F \cup G$, i.e., it suffices to prove the lemma in case $F=G=\varnothing$. By (2.2) $\operatorname{dim}\left(E \cap\left(R^{2} \times\{t\}\right)\right) \leqq 0$ for each $t \in R^{p-1}$, so (see $\left(^{*}\right)$ ) it suffices to prove the lemma at the points of $R_{p-1}(f)-E$, i.e., to assume $E=\varnothing$. Thus $R_{p-1}(f)$ is a $p$-manifold (or is $\varnothing$ ) and $f \mid R_{p-1}(f)$ has rank $p-1$.

We now apply [2, (3.9)] to each component $\Gamma$ of $R_{p-1}(f)$. Since $f$ is open, each $k(\Gamma)$ is odd and $B_{f}$ is contained in the at most $(p-1)$ dimensional analytic set $A=\bigcup_{\Gamma} A(\Gamma)$. Let $\Delta \subset A$ be the $C$-analytic subset given by (2.2). We may take $A=X$, and the conclusion results.

\section{Proof of the theorem.}

THEOREM 3.1. ([3, (1.1) and (4.1)].) Let $f: M^{n} \rightarrow N^{p}$ be a $C^{3}$ open map with $p \geqq 1$, and let $\operatorname{dim}\left(B_{f} \cap f^{-1}(y)\right) \leqq 0$ for each $y \in N^{p}$. Then there is a closed set $X \subset M^{p+1}$ such that $\operatorname{dim} f(X) \leqq p-2$ and, for every $x \in M^{p+1}-X$, there is a natural number $d(x)$ with $f$ at $x$ locally topologically equivalent to the map

$$
\phi_{d(x)}: C \times R^{p-1} \longrightarrow R \times R^{p-1}
$$

defined by $\phi_{d(x)}\left(z, t_{1}, \cdots, t_{p-1}\right)=\left(\mathscr{R}\left(z^{d(x)}\right), t_{1}, \cdots, t_{p-1}\right)$.

Proof of (1.1) 3.2. Let $X=X(f)$ be the complement of the set on which $f$ has the desired structure; then $X \subset B_{f}$ is closed. We 
suppose that $\operatorname{dim} f(X) \geqq p-1$, and will obtain a contradiction.

Since $f$ is $C^{3}, \operatorname{dim}\left(f\left(R_{p-2}(f)\right)\right) \leqq p-2[1$, p. 156]. If, for every $x \in M^{p+1}-f^{-1}\left(f\left(R_{p-2}(f)\right)\right)$, there is an open neighborhood $U_{x} \subset M^{p+1}-$ $f^{-1}\left(f\left(R_{p-2}(f)\right)\right)$ of $x$ with $\bar{U}_{x}$ compact and $\operatorname{dim}\left(f\left(U_{x} \cap X\right)\right) \leqq p-2$, it follows from the fact that $\left\{U_{x}\right\}$ has a countable subcover that $\operatorname{dim}(f(X)) \leqq p-2$. Thus, there is an $\bar{x} \in M^{p+1}-f^{-1}\left(f\left(R_{p-2}(f)\right)\right)$ such that, for every open neighborhood $U \subset M^{p+1}-f^{-1}\left(f\left(R_{p-2}(f)\right)\right)$ of $\bar{x}$, $\operatorname{dim}(f(U \cap X)) \geqq p-1$.

By [1, p. 156, Layering Lemma] there are open neighborhoods $U$ of $\bar{x}$ and $V$ of $f(\bar{x})$ and $C^{r}$ diffeomorphisms $\sigma: R^{2} \times R^{p-1} \approx U$ and $\rho: V \approx R \times R^{p-1}$ such that $\rho \circ f \circ \sigma=g$ is a $C^{r}$ layer map and $\sigma(\bar{x})=$ $(0,0)$. Thus $\operatorname{dim} g(X(g)) \geqq p-1$. By (2.3) there is a closed set $Y \subset$ $R^{2} \times R^{p-1}$ such that $\operatorname{dim} g(Y) \leqq p-2$ and $\operatorname{dim}\left(\left(B_{g}-Y\right) \cap g^{-1}(y)\right) \leqq$ 0 for each $y \in R \times R^{p-1}$.

Let $h$ be the restriction $g \mid\left[\left(R^{2} \times R^{p-1}\right)-Y\right]$; then $X(h)=X(g)-$ $Y, \operatorname{dim} h(X(h))=p-1$, and $\operatorname{dim}\left(B_{h} \cap h^{-1}(y)\right) \leqq 0$ for each $y \in R \times$ $R^{p-1}$, contradicting (3.1).

Examples 3.3. Open maps $f: M^{2} \rightarrow R$ with $\operatorname{dim}\left(B_{f} \cap f^{-1}(y)\right)=1$ are given in [4, p. 341] and [6, p. 329]; the latter example may be assumed to be $C^{\infty}$ except on one point inverse, and thus [1, p. 151] may be assumed to be $C^{\infty}$. As a result, " $f$ real analytic" may not be replaced by " $f C^{\infty}$ " in (1.1).

The maps $f$ and $g$ defined by $f(z)=\mathscr{R}(z)$ and $g(z)=(\mathscr{R}(z))^{3}$ are locally topologically equivalent at 0 , but are not locally $C^{1}$ equivalent, since $g$ has rank 0 at the origin.

There are examples $[2,(4.7)(b)]$ with $X=B_{f}, \operatorname{dim} B_{f}=p-1$, and $\operatorname{dim} f\left(B_{f}\right)=p-2$.

REMARK 3.4. A real analytic open map $f: M^{p} \rightarrow N^{p}$ is light [2, p. $28,(4.2)]$, and thus for $p \geqq 2$ satisfies a structure theorem $[1, \mathrm{p}$. 155] similar to (1.1).

\section{REFERENCES}

1. P. T. Church, Differentiable monotone mappings and open mappings, pp. 145-183 in The Proceedings of the First Conference on Monotone Mappings and Open Mappings, edited by L. F. McAuley, Oct. 8-11, 1970, SUNY at Binghamton, Binghamton, N. Y., 1971.

2. P. T. Church and W. D. Nathan, Real analytic maps on manifolds, J. Math. Mech., 19 (1969), 19-36.

3. P. T. Church and J. G. Timourian, Differentiable open maps of $(p+1)$-manifolds to p-manifold, Pacific J. Math., 48 (1973), 35-45. 
4. W. G. Fox, The critical points of peano interior functions defined on 2-manifolds, Trans. Amer. Math. Soc., 83 (1956), 338-370.

5. R. Narasimhan, Introduction to the Theory of Analytic Spaces, Lecture Notes in Mathematics No. 25, Springer Verlag, Berlin, 1966.

6. W. D. Nathan, Open mappings into a 1-manifold, pp. 322-342 in The Proceedings of the First Conference on Monotone Mappings and Open Mappings, edited by L. F. McAuley, Oct. 8-11, 1970, SUNY at Binghamton, Binghamton, N. Y., 1971.

7. - Open mappings on 2-manifolds, Pacific J. Math., (to appear).

8. H. Whitney, Tangents to an analytic variety, Ann. of Math., (2) 81 (1965), 495-549. 9. W. D. Nathan and F. Bruhat, Quelques proprietes fondamentales des ensembles analytiques-réels, Comment. Math. Helv., 33 (1959), 132-160.

Received August 21, 1972. Work of the first author supported in part by N. S. F. Grant GP-6871, and that of the second author by N. S. F. Grant GP-8888 and N. R. C. Grant A7357.

SYRACUSE UNIVERSITY

AND

UNIVERSITY OF ALBERTA 


\section{PACIFIC JOURNAL OF MATHEMATICS}

\section{EDITORS}

RICHARD ARENS (Managing Editor) University of California

Los Angeles, California 90024

R. A. BeaUmont

University of Washington Seattle, Washington 98105
J. DUGUNDJI*

Department of Mathematics University of Southern California Los Angeles, California 90007

D. Gilbarg and J. Milgram Stanford University

Stanford, California 94305

\section{ASSOCIATE EDITORS}

E. F. BECKENBACH

B. H. NeUmanN

F. WOLF

K. YoSHIDA

\section{SUPPORTING INSTITUTIONS}

\section{UNIVERSITY OF BRITISH COLUMBIA CALIFORNIA INSTITUTE OF TECHNOLOGY UNIVERSITY OF CA.LIFORNIA MONTANA STATE UNIVERSITY UNIVERSITY OF NEVADA NEW MEXICO STATE UNIVERSITY OREGON STATE UNIVERSITY UNIVERSITY OF OREGON OSAKA UNIVERSITY}

UNIVERSITY OF SOUTHERN CALIFORNIA STANFORD UNIVERSITY UNIVERSITY OF TOKYO UNIVERSITY OF UTAH WASHINGTON STATE UNIVERSITY UNIVERSITY OF WASHINGTON AMERICAN MATHEMATICAL SOCIETY NAVAL WEAPONS CENTER

* C. R. DePrima California Institute of Technology, Pasadena, CA 91109, will replace J. Dugundji until August 1974. 


\section{Pacific Journal of Mathematics}

\section{Vol. 50, No. $1 \quad$ September, 1974}

Gail Atneosen, Sierpinski curves in finite 2-complexes.............. 1

Bruce Alan Barnes, Representations of $B^{*}$-algebras on Banach spaces .... 7

George Benke, On the hypergroup structure of central $\Lambda(p)$ sets ....... 19

Carlos R. Borges, Absolute extensor spaces: a correction and an

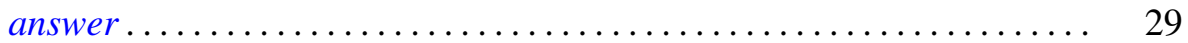

Tim G. Brook, Local limits and tripleability .................. 31

Philip Throop Church and James Timourian, Real analytic open maps .... 37

Timothy V. Fossum, The center of a simple algebra ............... 43

Richard Freiman, Homeomorphisms of long circles without periodic

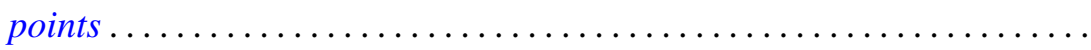

B. E. Fullbright, Intersectional properties of certain families of compact

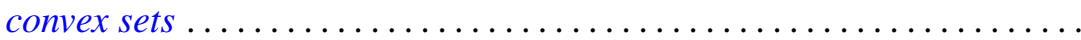

Harvey Charles Greenwald, Lipschitz spaces on the surface of the unit

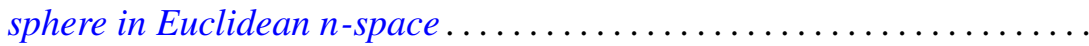

Herbert Paul Halpern, Open projections and Borel structures for

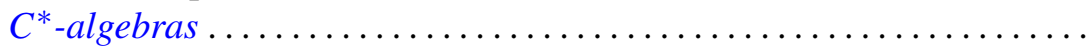

Frederic Timothy Howard, The numer of multinomial coefficients divisible

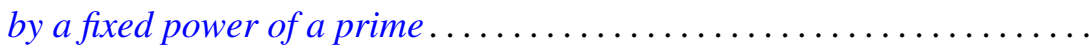

Lawrence Stanislaus Husch, Jr. and Ping-Fun Lam, Homeomorphisms of manifolds with zero-dimensional sets of nonwandering points........ 109

Joseph Edmund Kist, Two characterizations of commutative Baer rings ...

Lynn McLinden, An extension of Fenchel's duality theorem to saddle functions and dual minimax problems ................

Leo Sario and Cecilia Wang, Counterexamples in the biharmonic classification of Riemannian 2-manifolds...

Saharon Shelah, The Hanf number of omitting complete types ...

Richard Staum, The algebra of bounded continuous functions into a

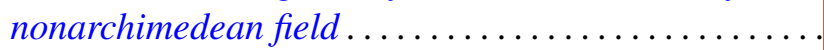

James DeWitt Stein, Some aspects of automatic continuity ..

Tommy Kay Teague, On the Engel margin

John Griggs Thompson, Nonsolvable finite groups all of whose local subgroups are solvable, $V \ldots \ldots \ldots \ldots \ldots \ldots \ldots \ldots$

Kung-Wei Yang, Isomorphisms of group extensions 